\title{
GRS 1758-258: RXTE Monitoring of a Rare Persistent Hard State Black Hole
}

\author{
M. Obst, K. Pottschmidt, A. Lohfink, J. Wilms, \\ M. Böck, D. M. Smith, J. A. Tomsick, I. Kreykenbohm
}

\begin{abstract}
GRS 1758-258 is the least studied of the three persistent black hole X-ray binaries in our Galaxy. It is also one of only two known black hole candidates, including all black hole transients, which shows a decrease of its $3-10 \mathrm{keV}$ flux when entering the thermally dominated soft state, rather than an increase.

We present the spectral evolution of GRS 1758-258 from RXTE-PCA observations spanning a time of about 11 years from 1996 to 2007. During this time, seven dim soft states are detected. We also consider INTEGRAL monitoring observations of the source and compare the long-term behavior to that of the bright persistent black hole X-ray binary Cygnus X-1. We discuss the observed state transitions in the light of physical scenarios for black hole transitions.
\end{abstract}

Keywords: black hole binaries: general, black hole binaries: individual: GRS 1758-258.

\section{Introduction}

GRS 1758-258 (Figure 1) is an intermediate mass Xray binary harboring a black hole and a companion consistent with an early A-type main sequence star, but with unusual colors [2]. Mass transfer is probably driven by Roche lobe overflow. Among such systems, usually transients, GRS $1758-258$ is one of only few persistent sources. Generally it can be found in the hard state. However, in some respects it still displays a behavior typical for transient sources [3-6, hysteresis, rare decay-type soft states].

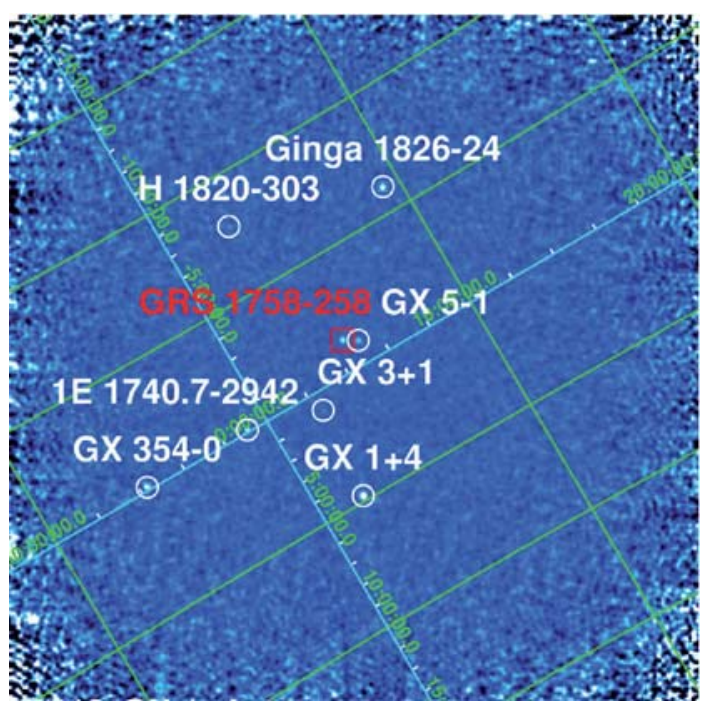

Fig. 1: INTEGRAL-ISGRI count rate mosaic image in the $20-40 \mathrm{keV}$ band obtained during Galactic Center Region Key Programme observations performed in spring 2007 [1]

\section{$2 \quad R X T E$ Monitoring}

GRS $1758-258$ was monitored by $R X T E$ in $1-1.5 \mathrm{ks}$ pointed snapshots monthly in 1996, weekly through 2000 and twice a week from March 2001 to October 2007. Every year there is a gap from November to January as the sun is too close to the Galactic center and RXTE cannot observe the source. The spectra were modeled taking into account the Galactic ridge background (see Sect. 2.1 for details). The flux has been corrected for the contribution of the Galactic ridge emission (Figure 2 top). Error bars are shown, typical errors are in the range of $1.0-1.5 \%$.

The photon index varies between 1.5 and 3 . Most of the time, GRS 1758-258 is in the hard state. However, seven dim soft states, during which the flux decreases and the spectrum softens, appear clearly in the data (Figure 2 bottom). However, no periodicity is detected in the occurrences of such soft states. During the 2001 soft state (highlighted in dark red $\triangle$ ), the source almost turned off completely. This strong decline in flux makes GRS 1758-258 especially interesting as it is typical for transient, not for persistent sources (see also hardness intensity diagram, Sect. 3).

\subsection{Background Modeling}

As GRS 1758-258 is a rather faint source located close to the Galactic Center (Figure 1), the RXTEPCA spectra contain not only source counts but also a strong background component caused by the Galactic ridge emission (Figure 3). In order to distinguish between these, a $13 \mathrm{ks}$ background ob- 


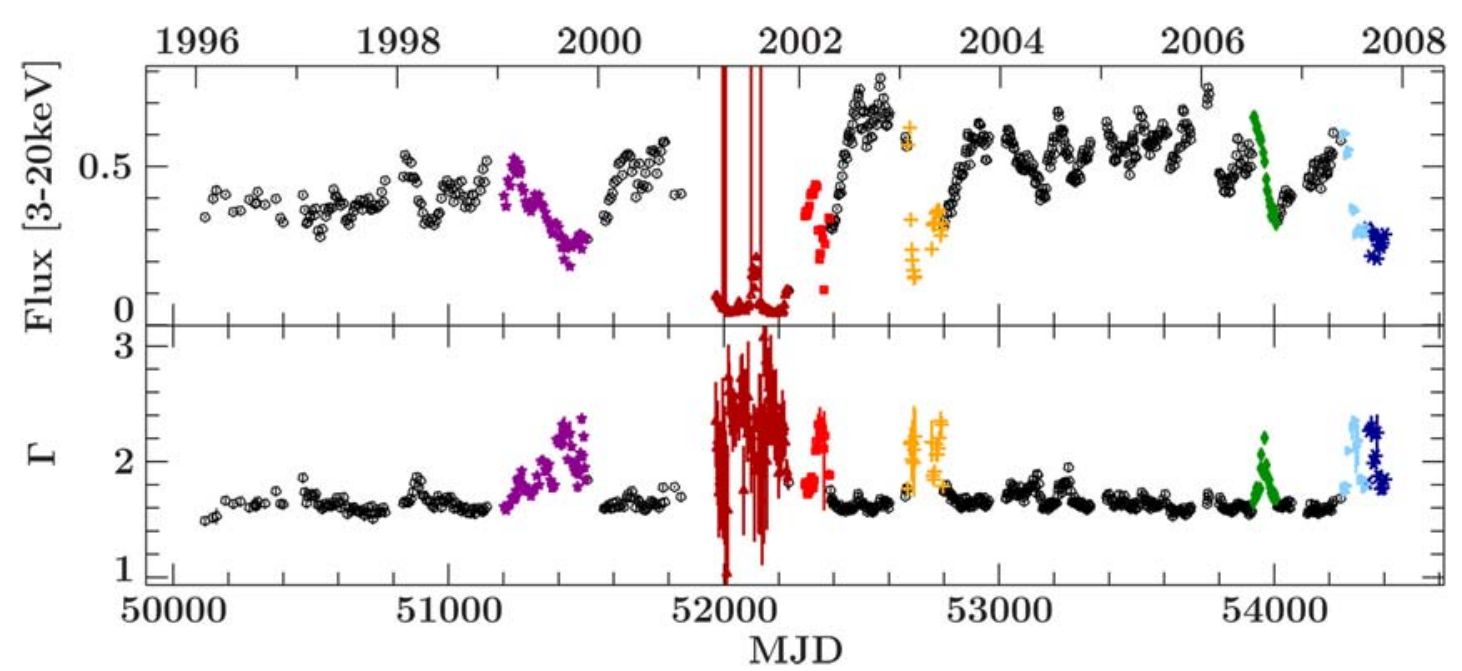

Fig. 2: Top: Flux in $\mathrm{keV} \mathrm{s}^{-1} \mathrm{~cm}^{-2}$ in the $3-20 \mathrm{keV}$ band, fitted to the spectra taken by RXTE. Bottom: Photon index obtained from modeling. Soft states are highlighted for episodes reaching a photon index above 2

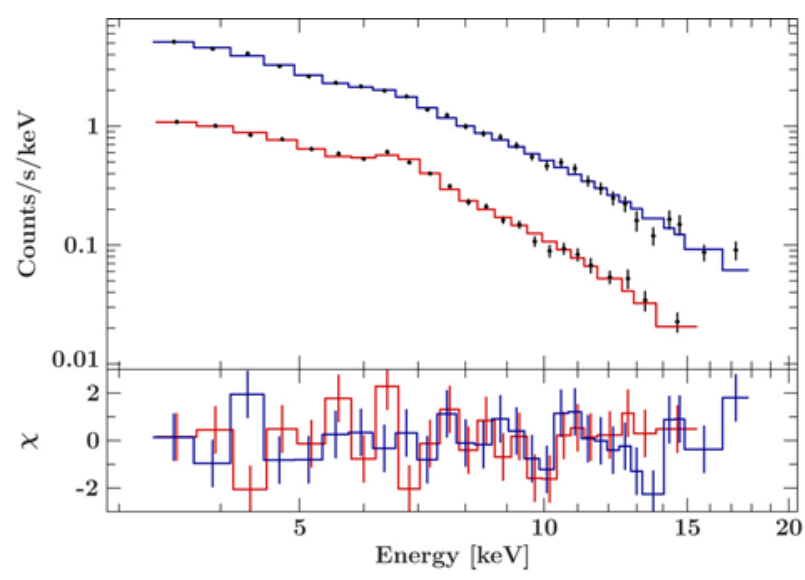

Fig. 3: Spectrum for the April 2003 GRS 1758-258 observation, i.e. containing the source as well as the Galactic ridge contribution (blue (black)), and spectrum for the Galactic ridge emission alone (red (grey))

servation $1.5^{\circ}$ offset from GRS 1758-258 was performed by RXTE in 1999. Figure 4 contains the spectrum modeled with two bremsstrahlung components $\left(F_{1,3-8 \mathrm{keV}}=0.015 \mathrm{keV} \mathrm{s}^{-1} \mathrm{~cm}^{-2}, k T_{1}=8 \mathrm{keV}\right.$, $\left.F_{2,3-8}=0.0027 \mathrm{keV} \mathrm{s}^{-1} \mathrm{~cm}^{-2}, k T_{2}=1.2 \mathrm{keV}\right)$. The iron line complex was modeled according to Galactic ridge observations performed with Suzaku [7]: Three lines at $6.4 \mathrm{keV}, 6.67 \mathrm{keV}$ and $7 \mathrm{keV}$, respectively, have equivalent widths that scale as 85:458:129.

\subsection{Spectral parameters}

The RXTE-PCA spectra in the $3-20 \mathrm{keV}$ band were fitted with an absorbed powerlaw, a weak neutral iron $\mathrm{K} \alpha$ line, and a black body disk component where required, always including the Galactic ridge emission (see Sect.2.1). The column density due to interstellar absorption in the direction of GRS 1758-258 is fixed at $N_{\mathrm{H}}=1.5 \times 10^{22} \mathrm{~cm}^{-2}$ according to earlier results [8]. An example is shown in Figure 5. The

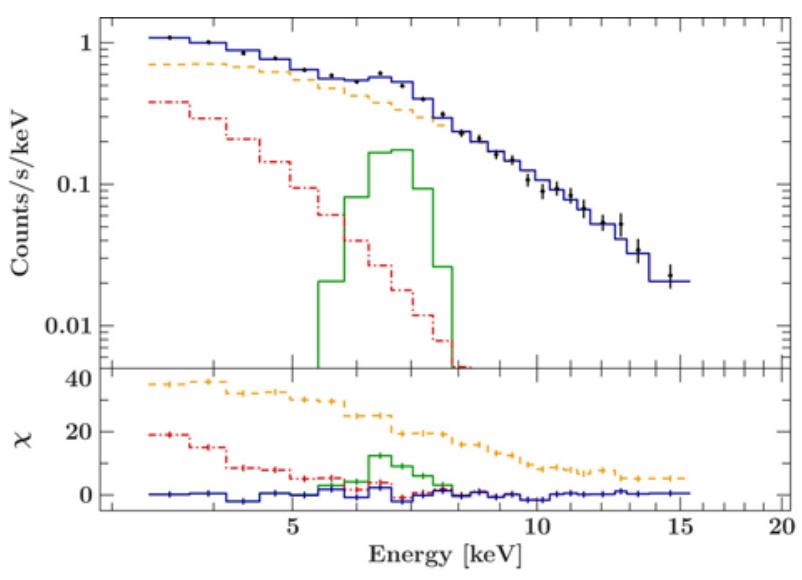

Fig. 4: Spectrum of the Galactic Ridge emission as seen by RXTE. The data were fitted with two bremsstrahlung components (1: orange dashed line, 2: red dash-dotted line) and an iron line complex as described in [7]

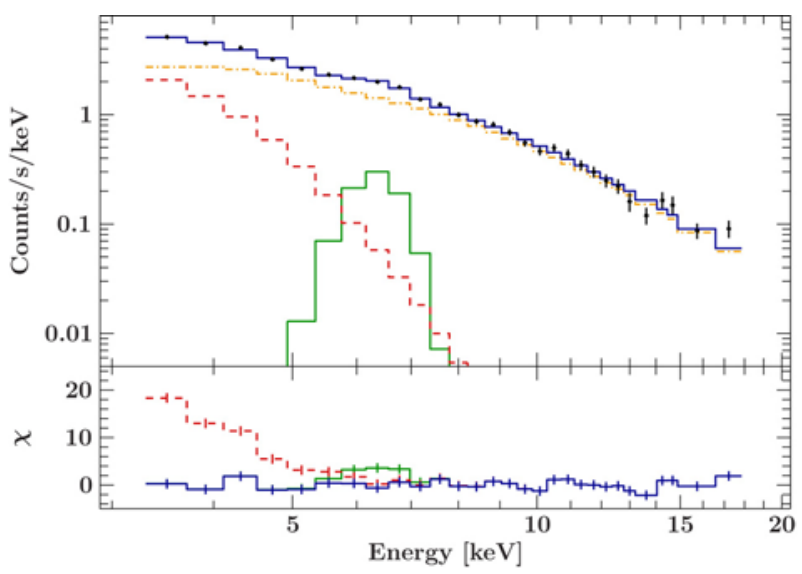

Fig. 5: Example spectrum taken by RXTE on 2009 April 08 , containing the absorbed powerlaw component (orange dash-dotted line), the disk (red dashed line) and the iron line (green (grey) solid line) 


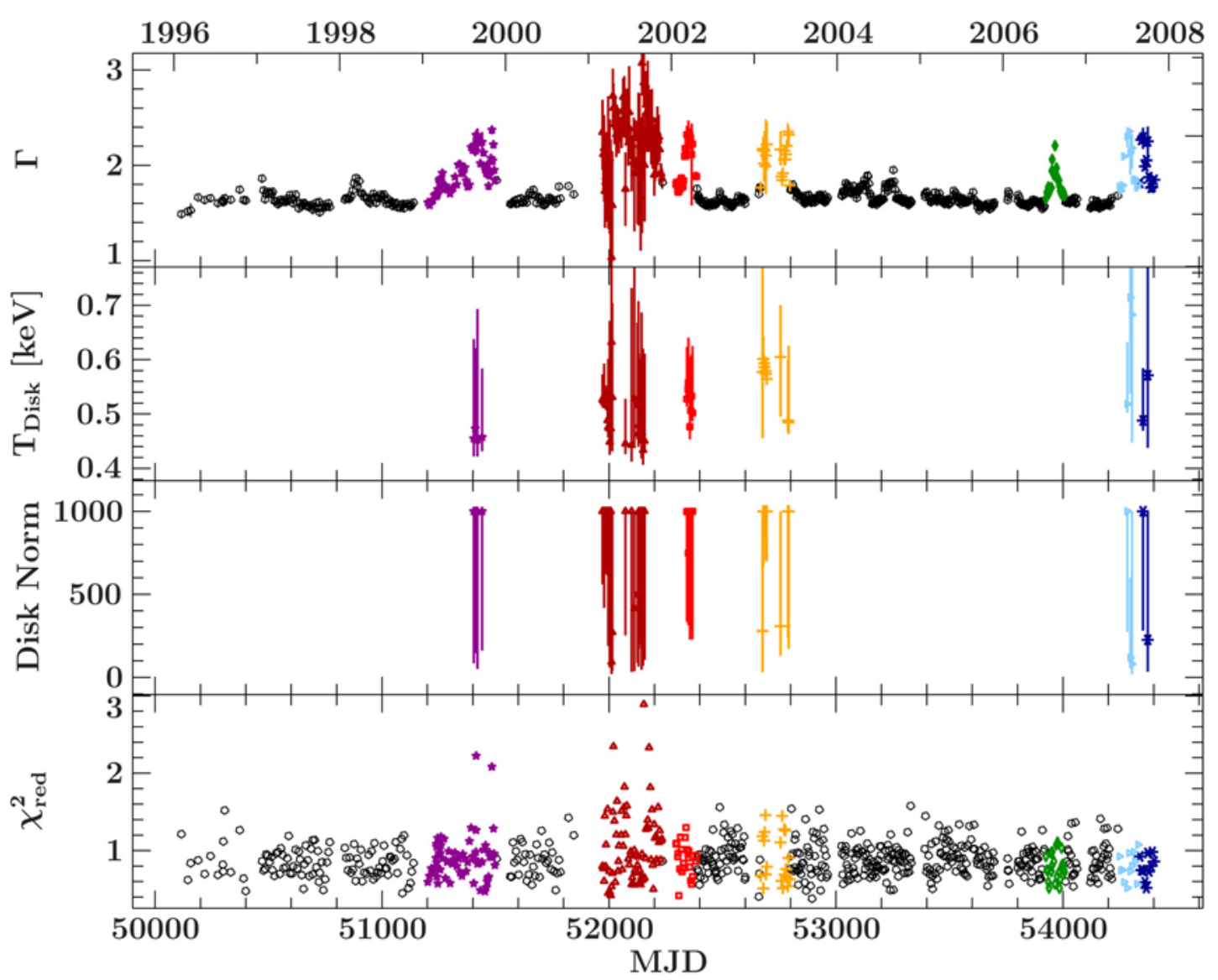

Fig. 6: Spectral parameters from RXTE monitoring observations of GRS1758-258: The photon index, temperature and normalization of the disk component, and the reduced $\chi^{2}$

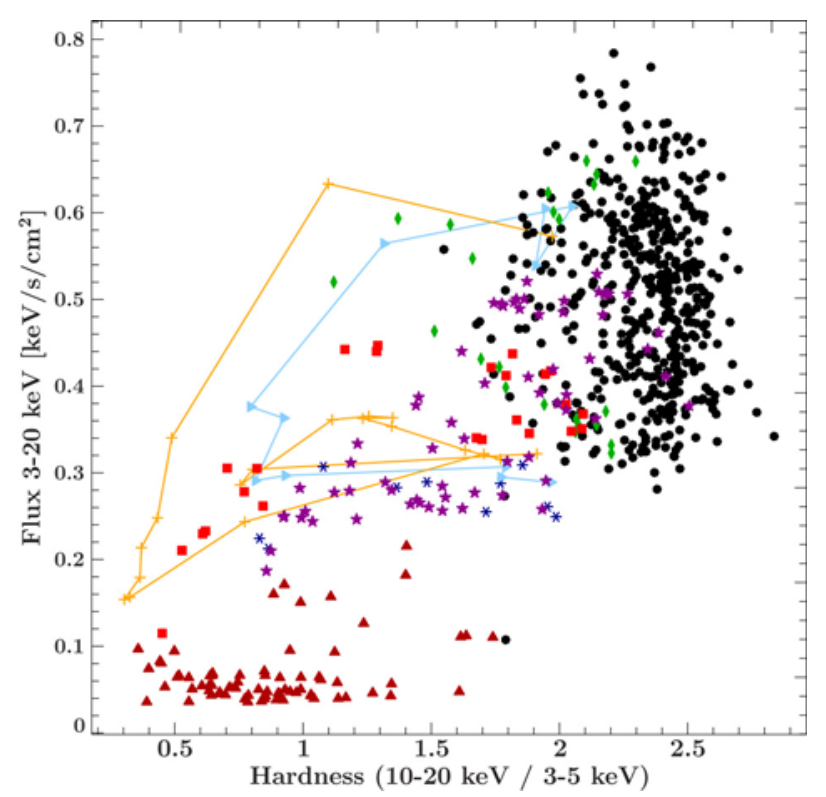

Fig. 7: Hardness intensity diagram (HID) from RXTE monitoring observations of GRS 1758-258 from 1997 to 2007. The seven dim soft states are highlighted as in Figures 6 and 2 disk becomes visible in the dim soft states, the low source flux increasing the error bars (Figure 6).

\section{Hardness intensity diagram}

For energies $<20 \mathrm{keV}$ the hardness intensity diagram (HID) of GRS 1758-258 shows a clear hysteresis for hard and soft state fluxes (absorbed fluxes, see Figure 7). This behavior is similar to that shown by black hole transients over their outbursts [9, q-shaped HID]. Different from transients, there is no rise in the hard state from quiescence. During the most extreme soft state the $3-20 \mathrm{keV}$ flux is clearly below the lowest hard state flux, with no full return to the hard branch observed down to near-quiescence. A comparison at these energies with our long-term RXTE monitoring observations of the persistent black hole $\mathrm{X}$-ray binary Cyg X-1 is in preparation.

Results from spectral fits to 2003-2009 INTEGRAL monitoring data of GRS 1758-258 [1] allow us to extend the HID studies to higher energies (Figure 8). As expected, neither source shows hysteresis for energies $>20 \mathrm{keV}$, i.e., in an energy range where only one, namely the hard, spectral compo- 


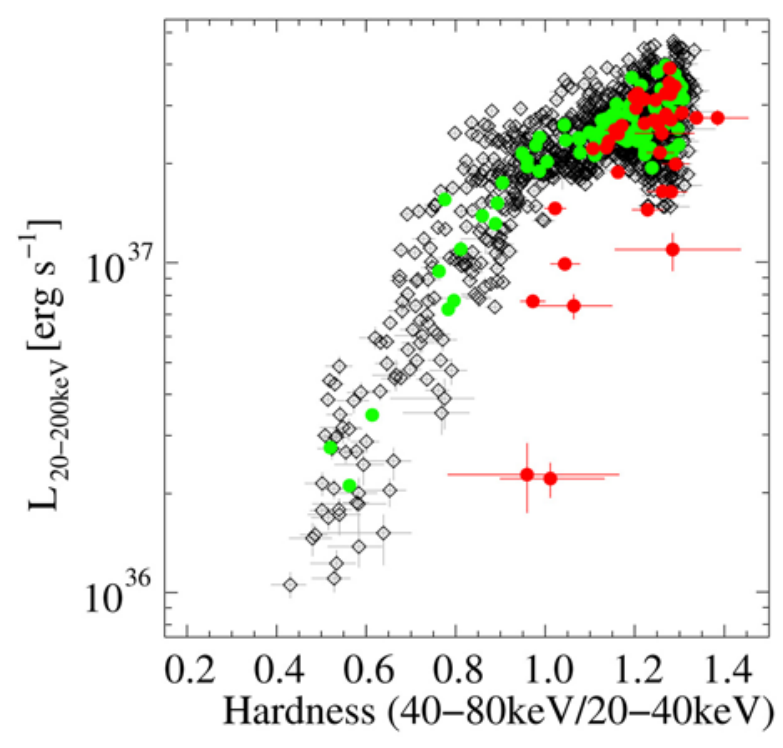

Fig. 8: HID from INTEGRAL monitoring of GRS 1758-258 from 2003 to 2009 (red (dark grey): monthly binning), compared to the HID from RXTE monitoring of Cyg X-1 from 1998 to 2010 (black: individual pointings, green (light grey): monthly binning)

nent dominates. Overall, the tracks of GRS 1758-258 in both HIDs are consistent with a persistent hard state source with occasional softening due to a temporary decrease in the mass accretion rate as suggested by [5]. The hard state HIDs of both sources show a remarkably similar range of hardnesses. Assuming a distance of $1.9 \mathrm{kpc}$ for Cyg X-1 as recently determined from radio parallax [10] and dust scattering halo measurements [11], the $>20 \mathrm{keV}$ luminosities of both sources would be similar if GRS 1758-258 had a distance of $6.5 \mathrm{kpc}$. Note, however, that while the decay towards softer, lower luminosity states is qualitatively similar in both sources as well, the luminosity of GRS 1758-258 has dropped more severely at a given hardness level than that of Cyg X-1.

\section{Summary and outlook}

The analysis of the 1996-2007 RXTE data of GRS 1758-258 reveals many details about the longterm behavior of the source. Typical hard state fluxes after taking into account the Galactic ridge emission are $0.2-0.4 \mathrm{keV} \mathrm{s}^{-1} \mathrm{~cm}^{-2}$. The spectra can be well described with an absorbed power law with a photon index of $1.5-3$. There are indications for a possible detection of an additional weak Fe line.

During the monitoring, there are 7 occurrences of soft states with photon indices softer than 2 . These states are most likely due to a decrease in the mass accretion rate. During the soft states, a marginal disk detection with $\mathrm{kT}_{\text {in }} \sim 500-800 \mathrm{eV}$ can be seen.
The $<20 \mathrm{keV}$ HID shows hysteresis with no full return to the hard branch observed down to nearquiescence. The $>20 \mathrm{keV}$ HID shows the same hard state luminosity and hardness as Cyg X-1 but a different decay.

\section{Acknowledgement}

This research has been partly funded by the European Commission under contract ITN 215212 "Black Hole Universe" and by DLR contract DLR50OR1007. K. Pottschmidt and A. M. Lohfink acknowledge support by NASA INTEGRAL GO grant NNX08AY17G and in addition A. M. Lohfink acknowledges support by the DAAD.

\section{References}

[1] Lohfink, A. M., Pottschmidt, K., Wilms, J., Lubiński, P.: ApJ, 2011, in prep.

[2] Muñoz-Arjonilla, A. J., Martí, J., LuqueEscamilla, P. L., et al.: $A \& \mathcal{E} A, 519, \mathrm{~A} 15,2010$.

[3] Pottschmidt, K., Chernyakova, M., Zdziarski, A. A., et al.: $A \& \mathcal{E} A, 452, \mathbf{2 8 5}, 2006$.

[4] Smith, D. M., Heindl, W. A., Markwardt, C. B., Swank, J. H.: ApJ, 554, L41, 2001.

[5] Smith, D. M., Heindl, W. A., Swank, J. H.: ApJ, 569, 362, 2002.

[6] Soria, R., Broderick, J. W., Hao, J., Hannikainen, D. C., et al.: $M N R A S, 2011$, in press (arXiv:1103.3009).

[7] Ebisawa, K., Yamauchi, S., Tanaka, Y., et al.: Progress of Theoretical Physics Supplement, 169, 121, 2007.

[8] Pottschmidt, K., Chernyakova, M., Lubiński, P., et al.: In Proc. 7th INTEGRAL Workshop. PoS, 2008, p. 98.

[9] Fender, R. P., Belloni, T. M., Gallo, E.: $M N-$ $R A S, 355, \mathbf{1}$ 105, 2004.

[10] Reid, M. J., McClintock, J. E., Narayan, R., et al.: 2011, arXiv:1106.3688.

[11] Xiang, J., Lee, J. C., Nowak, M. A., Wilms, J.: ApJ, 2011, in press (arXiv:1106.3378).

M. Obst

Remeis Observatory/ECAP

Bamberg, Germany 
K. Pottschmidt CRESST/NASA-GSFC

Greenbelt, MD, USA

UMBC

Baltimore, MD, USA

A. Lohfink
UMCP
College Park, MD, USA

J. Wilms

Remeis Observatory/ECAP

Bamberg, Germany

M. Böck

Remeis Observatory/ECAP

Bamberg, Germany
D. M. Smith SCIPP/UCSC

Santa Cruz, CA,USA

J. A. Tomsick

SSL/UCB

Berkeley, CA, USA

I. Kreykenbohm

Remeis Observatory/ECAP

Bamberg, Germany 